\title{
Application Limits of the Ferrioxalate Actinometer
}

\author{
Benjamin Wriedt* Prof. Dr. Dirk Ziegenbalg ${ }^{\text {* }}$
}

May 30, 2021

\begin{abstract}
Evaluating the efficiency of newly designed photoreactors is crucial for systematic development and optimization of photochemical processes. A suitable tool is actinometry, prominently represented by the most widely studied and applied ferrioxalate system. However, such measurements show reproducible problems in the data consistency. This study scrutinizes these issues and approaches an experimental elucidation. An application limit for the ferrioxalate actinometer under intense irradiation was identified and experimentally validated. A drop of the quantum yield at high incident photon fluxes, generating high local concentrations of carboxyl radicals, leads to systematically wrong measurements. For reliable measurements with the ferrioxalate actinometry, a continuous operation mode or extensive mixing should be ensured.
\end{abstract}

\section{Introduction}

The symbiosis of microreaction technology and continuous reaction control has proven to be a promising concept to increase the efficiency of photoreactors. [1]5] Continuous flow reactors also allow for modular parallelization when transferred to a larger production scale, also called numbering up. [6] For a systematic development of efficient photoreactors, a measure is needed to evaluate and compare the general performance. An essential parameter for that is the amount of photons available within the reaction volume during irradiation time.

The photon flux inside a photoreactor can be determined by chemical actinometry, especially for milli- or micro-scale devices. 7$]$ This measurement technique utilizes a chemical reaction with well-known parameter-dependency that is conducted right within the reactor under investigation. The measurement takes place under the same conditions as the originally desired photoreaction. Consequently, the residence time and optical effects (transmission, reflection and scattering) are

\footnotetext{
*Ulm University, Institute of Chemical Engineering, Albert-Einstein-Allee 11, 89081 Ulm, Germany

$\dagger$ corresponding author
} 
intrinsically included. Based on the caused conversion and the incident light spectrum, the available photon fluxes can be calculated spectrally resolved. The received quantities are obtained in molecular and photonic units, so no transfer from energetic data (as if measured by physical devices) is necessary.

An overview of the most widely studied and thus recommended actinometers can be found in an article of the IUPAC Commission on Photochemistry by Kuhn et al. ${ }^{[8]}$ One of the most prominent actinometers is the ferrioxalate system introduced by Hatchard and Parker. It provides sensitivity in the wavelength range between 200 and $450 \mathrm{~nm}$ as well as simple handling. [9] Necessary prerequisites for reliable measurements are the independence of the wavelength of the incident light, temperature, concentration, possible impurities and atmospheric oxygen on the quantum yield. Application limits were defined by batch experiments with low-intensity light sources to meet these conditions. [9]

State-of-the-art devices, however, do not necessarily match these conditions. Within the last decades, a significant improvement of several process parameters has led to a comprehensive intensification of the irradiation conditions inside these new photoreactors. Decisive influences for this are e.g. more efficient and powerful light sources, more sophisticated reactor geometries and dynamic process control. $\stackrel{[10][19]}{-}$ Along with this technical progress, actinometry is still used following the recommendations of Hatchard and Parker, even if some results showed severe inconsistencies. Clear signs of this proneness for measurement errors are the numerous adaptions and additional hints published in articles, books and reports. $20-26]$ The consequent association with a certain inaccuracy of measurements is a crucial drawback for the entire actinometric method.

This work aims for identification, characterization and avoidance of systematic error sources encountered in practical application of the ferrioxalate actinometer. Crucial factors causing inaccuracies are scanned experimentally and brought in line with recent spectroscopic findings, leading to recommendations for a reliable measurement workflow.

\section{Results and Discussion}

\subsection{General Aspects of Ferrioxalate Actinometry}

The Hatchard-Parker actinometer is based on the irreversible light-induced redox reaction of ferrioxalate. The central iron atom is converted from $\mathrm{Fe}^{\mathrm{III}}$ to $\mathrm{Fe}^{\mathrm{II}}$ according to the empirically found brutto equation:

$2\left[\mathrm{Fe}^{\mathrm{III}}\left(\mathrm{C}_{2} \mathrm{O}_{4}\right)_{3}\right]^{3-} \stackrel{h v}{\longrightarrow} 2\left[\mathrm{Fe}^{\mathrm{II}}\left(\mathrm{C}_{2} \mathrm{O}_{4}\right)_{2}\right]^{2-}+2 \mathrm{CO}_{2}+\mathrm{C}_{2} \mathrm{O}_{4}{ }^{2-}$

After irradiation, $\mathrm{Fe}^{\mathrm{II}}$ is quantitatively complexed by adding a 1,10-phenanthroline solution to form the deeply red ferroin. The conversion can be determined by pho- 
tospectrometry and a comprehensive calculation finally yields the incident photon flux. [20]

Motivated by the inconsistencies outlined above, time-resolved FT infrared spectroscopic investigations were conducted to identify the reaction mechanism in detail. 27. 29] In the first photochemical step, a highly excited carbon dioxide molecule is split off from one of the oxalate ligands. A carboxylate radical anion $\mathrm{CO}_{2}{ }^{\bullet-}$ remains at the iron center as an oxygen-coordinated ligand (reaction $\mathrm{R}$ 2.2 . [29]

$$
\left[\mathrm{Fe}^{\mathrm{III}}\left(\mathrm{C}_{2} \mathrm{O}_{4}\right)_{3}\right]^{3-} \underset{2.0 \cdot 10^{12} \mathrm{~s}^{-1}}{\longrightarrow}\left[\mathrm{Fe}^{\mathrm{II}}\left(\mathrm{C}_{2} \mathrm{O}_{4}\right)_{2}\left(-\mathrm{OC}^{\bullet} \mathrm{O}\right)\right]^{3-} \cdot \mathrm{CO}_{2}
$$

The carboxyl radical is then cleaved off from the metal complex (reaction $\mathrm{R}$ 2.3 . In the presence of neutral $\mathrm{CO}_{2}$, the carboxyl radical is in equilibrium with the oxalyl radical $\left(\mathrm{C}_{2} \mathrm{O}_{4}\right)^{\bullet-}$ (equation $\mathrm{R} 2.4$.

$$
\begin{aligned}
{\left[\mathrm{Fe}^{\mathrm{II}}\left(\mathrm{C}_{2} \mathrm{O}_{4}\right)_{2}\left(-\mathrm{OC}^{\bullet} \mathrm{O}\right)\right]^{3-} \cdot \mathrm{CO}_{2} } & \stackrel{2.5 \cdot 10^{9} \mathrm{~s}^{-1}}{\longrightarrow}\left[\mathrm{Fe}^{\mathrm{II}}\left(\mathrm{C}_{2} \mathrm{O}_{4}\right)_{2}\right]^{2-}+\mathrm{OCO}^{\bullet-}-\mathrm{CO}_{2}\left(\mathrm{R}_{2}\right. \\
\mathrm{OCO}^{\bullet--} \cdot \mathrm{CO}_{2} & \rightleftharpoons\left(\mathrm{C}_{2} \mathrm{O}_{4}\right)^{\bullet-}
\end{aligned}
$$

Since on short time scales all three species of reaction $\mathrm{R} 2.4$ are present in close vicinity to the surrounding ferrioxalate complex, the carboxyl radical can also attack one more ferrioxalate complex. Cleaving off another ligand, an oxalate molecule and a carbon dioxide molecule can be formed (reaction R 2.5).

$\left[\mathrm{Fe}^{\mathrm{III}}\left(\mathrm{C}_{2} \mathrm{O}_{4}\right)_{3}\right]^{3-}+\left(\mathrm{CO}_{2}\right)^{\bullet-} \stackrel{2.9 \cdot 10^{5} \mathrm{~s}^{-1}}{\longrightarrow}\left[\mathrm{Fe}^{\mathrm{II}}\left(\mathrm{C}_{2} \mathrm{O}_{4}\right)_{2}\right]^{2-}+\left(\mathrm{C}_{2} \mathrm{O}_{4}\right)^{2-}+\mathrm{CO}_{2}(\mathrm{R} 2.5)$

Caused by this electron transfer to another metal complex, the quantum yield can increase up to 1.35. This value was already postulated in 1955 and could be measured experimentally in 1979 for wavelengths below $254 \mathrm{~nm} .30 .31]$

\subsection{Systematic Inaccuracies}

To overcome pitfalls leading to systematic errors during the measurements, a revised general working procedure was recently published. 32$]$ The procedure is split in three main parts. The preliminary considerations consist of a verification of the physical and chemical properties, data acquisition of the reactor setup, adjustments of the absorption fraction within the experimental limits and the actinometer preparation. In addition to the actual measurements, the practical block includes an unirradiated zero sample before and after the experiment to ensure that all conversion is only caused by irradiation in the photoreactor. As there is usually a small amount of product found prior to the experiment, this value is used as a baseline 


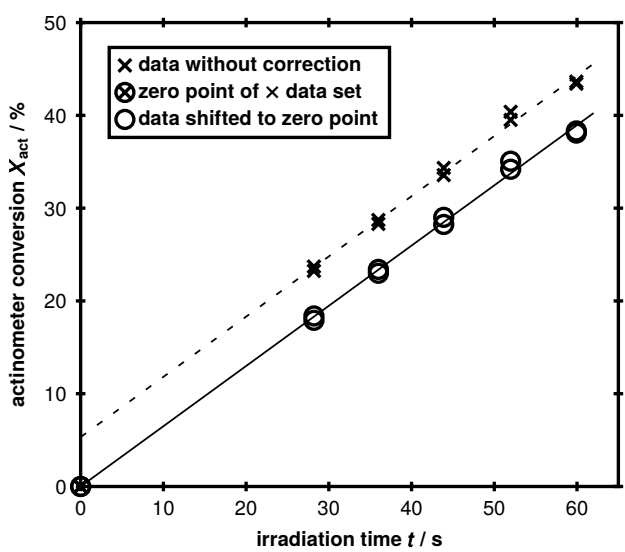

(a)

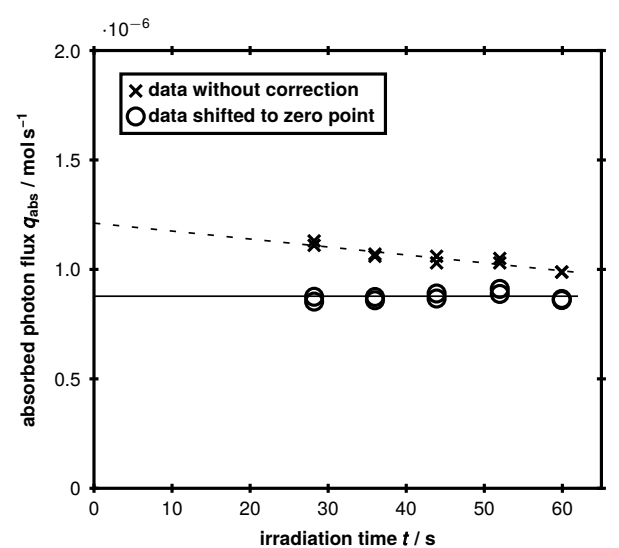

(b)

Figure 1: Example for the conversion $X_{\text {act }}$ (a) and the calculated photon fluxes $q_{\text {abs }}$ (b) from the raw data without correction and with the data shifted by the yintersection to make the regression line cross the zero point.

if it matches the value of the blind sample after the experiment. Additionally, a reference measurement in a simple, standardized irradiation setup is taken.

Mathematical evaluation is carried out considering both the actinometer system properties and the irradiation conditions to obtain the irradiated photon flux based on absorbance by the actinometer $q_{\mathrm{abs}}$. Provided that the procedure is carried out correctly, $q_{\text {abs }}$ calculated equals the incident photon flux $q_{\mathrm{p}}$. But despite strictly following these instructions and hints, some actinometer measurements systematically fail for specific photoreactor setups. No matter if e.g. mercury vapor lamps or LEDs at various wavelengths are used, no correct result can be obtained.

The relevant issue becomes visible when plotting the measured ferrioxalate conversion $X_{\text {act }}$ over the irradiation time $t$. This is illustrated in Figure 1a, where the experimental data obtained in a capillary reactor irradiated with a $405 \mathrm{~nm}$ LED is presented. In this reactor, the capillary is horizontally arranged to ensure a geometrical match of the emission characteristics of the LED and the capillary. The setup is shown in detail in the experimental section 4.1. The data for actinometer conversion has already been corrected for the unirradiated zero measurement.

Even though the correlation is linear, its extrapolation does not cross the diagram origin (dashed line). To make the effects of a disregard clearly comprehensible, a series of measurements with a particularly large y-axis intercept of over $5 \%$ was chosen for this example. In contradiction to the taken zero sample without any irradiation (shown at the origin), the conversion at an irradiation time of $0 \mathrm{~s}$ would not be $0 \%$. Justified by the reproducibility of the single measured values and the straight regression line, an insufficient amount of data points can be excluded.

Furthermore, this observation is not reactor-specific and can also be observed for different reactor concepts, albeit with other absolute values for the y-axis in- 
tercept. Also in literature, there are hints for this phenomenon in other published raw data. However, due to the lack of exact data, it cannot be verified whether a mathematical correction was implemented in the subsequent evaluation. [20|31|33|34|

The raw data can be corrected through shifting the absolute measurement by the value of the y-axis intersection of the regression line. As a result, the linear regression crosses the origin. On the one hand, this correction leads to a contenting result for the calculated photon flux as it is independent of the irradiation time (Figure 1b). If the uncorrected data were nevertheless evaluated, there is no definite result for $q_{\mathrm{abs}}$ as it depends on the irradiation time. On the other hand, this mathematical correction significantly alters the absolute value of the calculated photon flux. Considering the given example, the deviation is almost $30 \%$ at an irradiation time of $28 \mathrm{~s}$.

To systematically investigate the inaccuracy of the ferrioxalate actinometry for its reason, the self-made capillary reactor was extended to allow the installation of several capillary rows as example systems. Those 3D-printed reactor scaffolds only differ in the relative capillary arrangement according to Figure 2 and are irradiated with the same LED at $405 \mathrm{~nm}$. Measurements were conducted either while pumping the actinometer through the capillaries and subsequent recycling of the reaction solution (continuous operation) or while the flow was stopped (discontinuous operation). For all of these setups, a regression of the actinometer conversion yielded $\mathrm{y}$-axis intercepts unequal 0 when measurements were conducted for discontinuous operation.
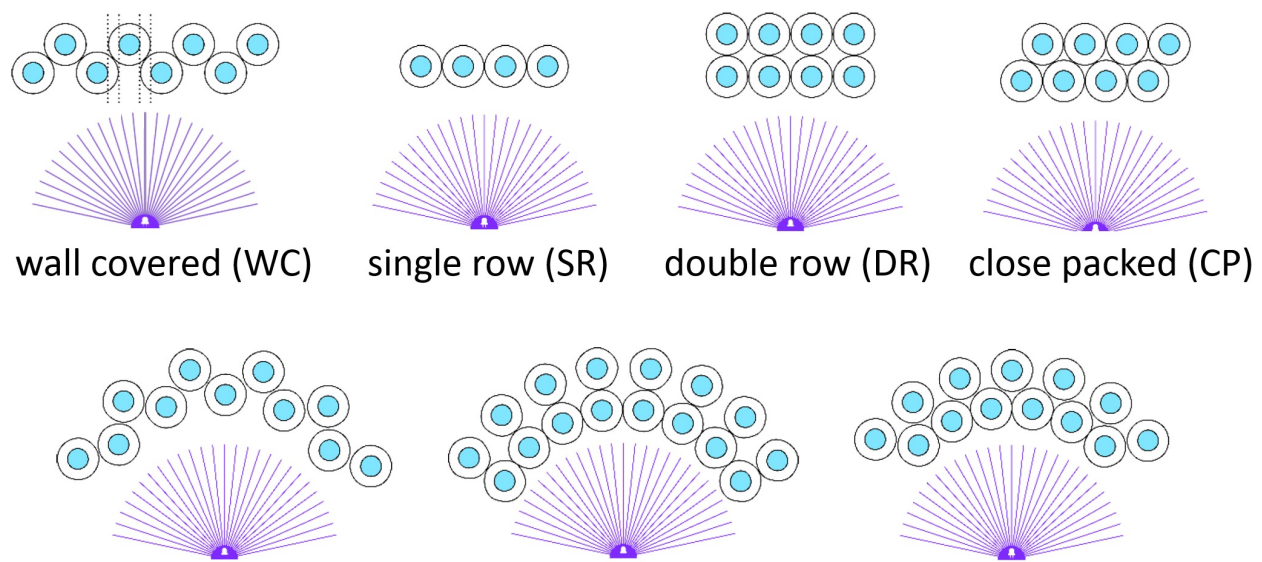

wall covered bent (WCB) double row bent (DRB) close packed bent (CPB)

Figure 2: Schematic overview of the cross-sectional arrangement within the applied capillary reactors.

Consequently, the deviation between continuous and discontinuous operation were elaborated. The results after the correction of the y-axis intercept are shown in Figure 3. For interpretation, the varying $q_{\mathrm{abs}}$ of the reactors are only considered individually without mutual comparison.

Although only differing by the operation mode, the measurements deviate sig- 


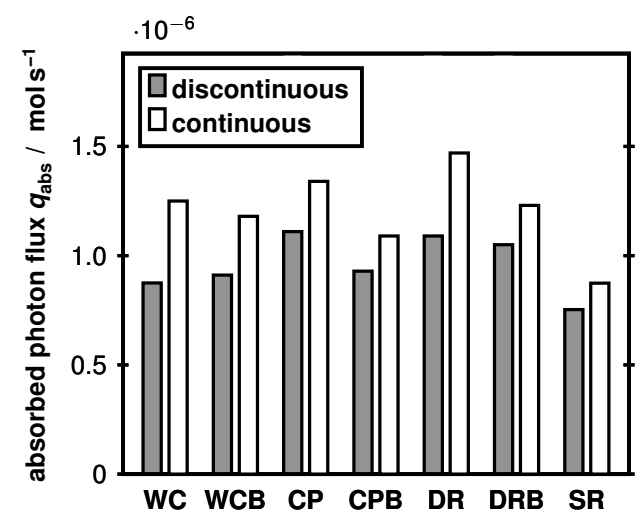

Figure 3: Measured photon fluxes for discontinuous and continuous ferrioxalate actinometry in different capillary reactors.

nificantly. The largest mismatch of $44 \%$ is reached in the WC-reactor, the other differences lie between 16 and $34 \%$. In view of these high values, it is noticeable that this inconsistency has not been observed or studied yet. The $q_{\mathrm{abs}}$ of the discontinuous measurements show higher and more fluctuating deviations. A major reason for this is that the corrected y-axis intercepts of the regression lines are much higher than for continuous measurements (1a). In consequence, the mismatch was therefore attributed to the discontinuous ferrioxalate measurements.

\subsection{Results and Discussion}

To identify the reasons for the observed inconsistencies, parameters that potentially influence the outcome of the actinometric measurements were investigated. Table 1 summarizes the relevant parameters and the followed test approach.

Table 1: List of the five most probable parameters that can cause the found discrepancy in the experimental data and the used approach to test the suspected cause.

\begin{tabular}{ll}
\hline Potential Parameter & Experimental Test \\
\hline 1. inaccurate physical parameters & verification of literature \\
2. thermal influence of the light source & step-wise irradiation \\
3. different flow conditions in the capillaries & different pumping speeds \\
4. light effects between capillary layers & different layer fillings \\
5. changes of quantum yield & variation of influence parameter \\
\hline
\end{tabular}

First, inaccurate physical parameters used in the mathematical evaluation can cause deviations. This concerns the wavelength-dependent absorption coefficients of the ferrioxalate system or the accuracy and range of the actinometer calibration. The measured absorption coefficient as well as the calibration were in agreement with literature (see SI, section S1]. ${ }^{8[9 \mid 35]}$ Furthermore, a potential influence of the 


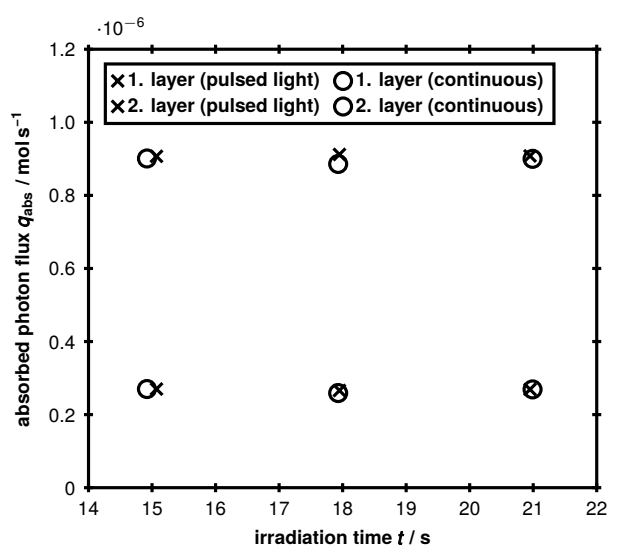

(a)

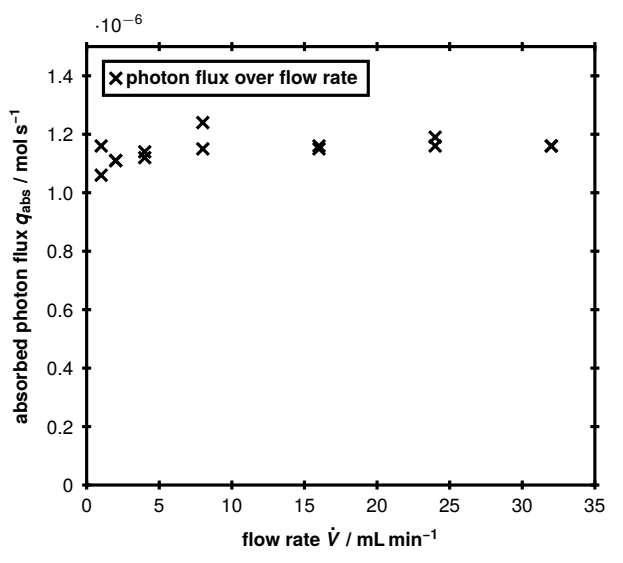

(b)

Figure 4: Experiments to check for the dependence on thermal influence of the light source (a) and back-mixing (b).

$\mathrm{pH}$ value at the applied experimental conditions could be ruled out according to a former investigation. ${ }^{32]}$ Consequently, this parameter is unlikely to cause the observed inconsistencies.

Temperature changes during the measurement can lead to an undesired formation of $\mathrm{Fe}^{\mathrm{II}}$ through a thermal reaction path in addition to the photochemical reaction. Heating can occur, for example, due to insufficient heat dissipation through the LED heat sink. [36] If temperatures of over $60^{\circ} \mathrm{C}$ are reached locally, the temperature range tested by Hatchard and Parker for reliable results would be exceeded. Thus, to rule out any influence of temperature on the photonic conversion, two test series were run with the same total irradiation time. In the first one, the LED was operated continuously. In the second one, every irradiation interval of only $1 \mathrm{~s}$ was followed by a pause of $1 \mathrm{~min}$ to give the system enough time to dissipate thermal energy. The results gained in a two layer capillary reactor for three different total irradiation times are shown in Figure 4a. Both series of measurements show completely identical results for both capillary layers. Thus, it can be excluded that a thermal influence causes additional conversion, resulting in a mistakenly calculated higher $q_{\text {abs }}$.

Different flow conditions cause differences in the concentration fields in the reactor, resulting in a local change of the absorption factor. For continuous operation, the flow rate alters the residence time of specific fluid elements. Figure $4 \mathrm{~b}$ indicates that from a flow rate of $8 \mathrm{~mL} \mathrm{~min}^{-1}$, the measured values remain constant. Thus, different local flow conditions are demonstrably not the reason for the deviation between continuous and discontinuous results. Since an insufficient volume of the recirculating actinometer solution can also lead to errors, changing this parameter was also investigated. An increase in the total circulated volume from $20 \mathrm{~mL}$ to $40 \mathrm{~mL}$ also had no effect at all, excluding insufficient mixing in the receiver vessel 


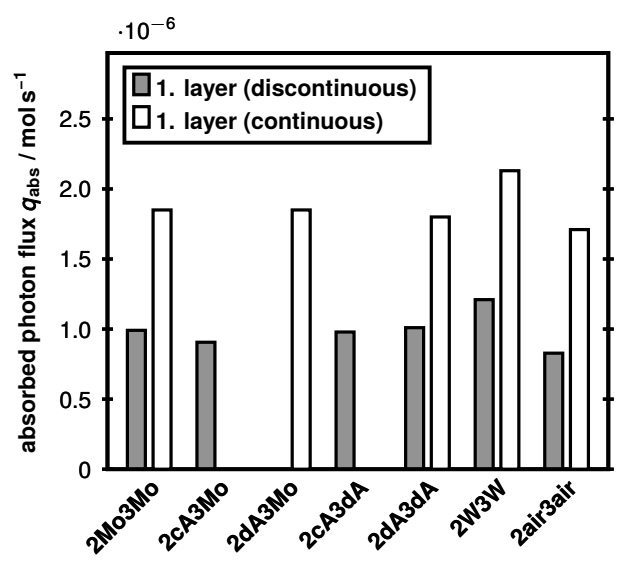

(a)

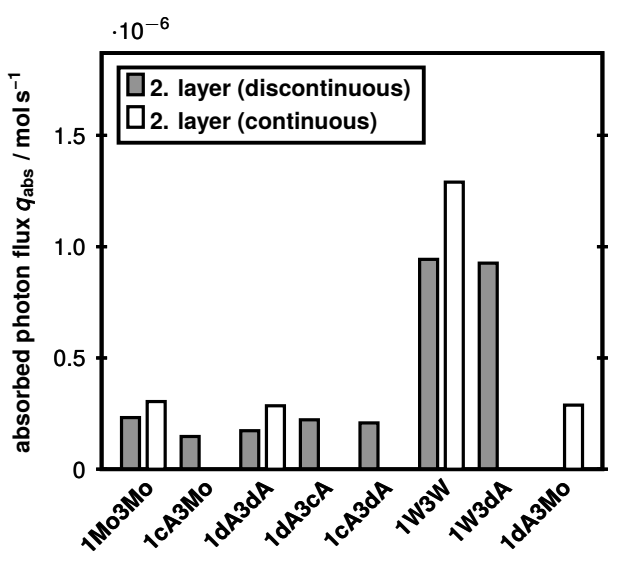

(b)

Figure 5: Actinometry at different conditions in the first (a) and second (b) capillary layer. (number $=$ capillary layer, $\mathrm{Mo}=$ methyl orange, $\mathrm{cA}=$ actinometer (continuous), $\mathrm{dA}=$ actinometer (discontinuous), $\mathrm{W}=$ water, air $=$ empty)

(separately shown in the SI, section $\mathbf{S 2}$.

Changes in the radiation field in the reactor can also play a role. These can be caused by optical effects such as reflection or scattering at multiple material borders between air, the irradiated capillary and the inner liquid. The reaction progress can also lead to varying absorption conditions in the capillaries. The impact of light effects depends mainly on the number of material borders crossed by irradiated photons. If several capillaries are arranged close to each other, the number of these passages increases many times over, making it much more difficult to follow the path of a single photon. Consequently, the study aimed to determine whether actinometer measurements are affected by differently filled capillaries of directly adjacent layers. For this purpose, continuous and discontinuous measurements were performed in a reactor extending the close packed (CP) layout by adding a third layer in the closest packed arrangement.

Single layers were actinometrically investigated by conducting continuous and discontinuous measurements. To simulate different radiation fields, the surrounding capillaries were subsequently filled with water, air, ferrioxalate solution or a methyl orange solution. Water and air were investigated for the impact of different refractive indices. The ferrioxalate solution simulates the typical setup for actinometric measurements and a methyl orange solution was chosen to absorb the incident light. For the chosen concentrations light is completely absorbed, simulating a degree of absorption of $f \approx 1$. Similar to the layer that was probed for the incident photon flux, the impact of the ferrioxalate solutions in the other layers was investigated for continuous and discontinuous operation.

Figure 5 shows $q_{\text {abs }}$ for the first (a) and second (b) capillary layers with continuous and discontinuous measurements. The transmission to the third layer was 
too low to obtain meaningful data. In the first layer, all discontinuous values for the absorbed photon flux ranged from $9.06 \cdot 10^{-7}$ to $1.01 \cdot 10^{-6} \mathrm{~mol} \mathrm{~s}^{-1}$, provided that actinometer solution or methyl orange was in the two layers behind the probed layer (experiments "2Mo3Mo" to "2dA3dA", see the Figure caption for the nomenclature). Within the limits of measurement accuracy, no influence of the operation mode or a decreasing absorbance in other layers can be observed. If the two capillary layers behind are filled with water or air, the different reflection conditions and refractive indices alter the $q_{\mathrm{abs}}$. With water, $q_{\mathrm{abs}}$ slightly increased by about $20 \%$. The reverse happens for empty capillaries, where a $17 \%$ lower $q_{\mathrm{abs}}$ is detected. The continuous measurements confirm the qualitative findings of the discontinuous measurement series. Independent of the other capillary layer filling, a constant $q_{\text {abs }}$ of about $1.82 \cdot 10^{-6} \mathrm{~mol} \mathrm{~s}^{-1}$ is determined. In combination with water, the value increases by $18 \%$, air-filled capillaries lead to a decrease by $8 \%$. However, the difference between the two operation modes is considerably high. In some cases, $q_{\mathrm{abs}}$ differs by more than $200 \%$ for individual measurements under the same irradiation conditions.

In comparison, for the second layer $q_{\mathrm{abs}}$ ranges from $1.73 \cdot 10^{-7}$ to $2.32 \cdot 10^{-7} \mathrm{~mol} \mathrm{~s}^{-1}$. Thus, the absolute deviation within the measurement series is only about half as large as in the first layer. In relative terms, with a deviation of $30 \%$ for $q_{\mathrm{abs}}$, the inconsistency is still high. In the case of a completely absorbing first layer, the results are independent of the filling with actinometer or methyl orange. The absorbed photon fluxes under a layer of water are considerably higher. It can be deduced that fully absorbing layers do not affect each other and also the decreasing degree of absorption in the investigated parameter space has no effect.

It can be concluded, that the observed inconsistencies can be reproduced but not influenced by specific operation conditions. Hence, the reason for the deviation might be ascribed to an alteration of the chemical reaction mechanism. Nevertheless, the application in an LED-irradiated microreactor must play a role, even if no such finding is mentioned nor explained in the literature of the past decades. $\frac{|89| 20|21| 37 \mid}{}$ This leaves a changing quantum yield as the last potential parameter, which can result from a changing reaction mechanism and also relates to the light source or with other words to the incident photon flux. As an appropriate experimental procedure for testing an alteration of the quantum yield is not trivial, spectroscopic studies are conflated with the investigations so far in the next section.

\subsection{Insights from Recent Spectroscopic Studies}

Several research groups agree with the original results of Hatchard and Parker that under conventional conditions for actinometry, the quantum yield $\Phi$ depends on the wavelength but is otherwise constant. $\frac{[37 \mid 38]}{1}$ In consecutive experiments, Goldstein and Rabani investigated the general dependence of the quantum yield at a ferrioxalate concentration lower than $0.006 \mathrm{M}$ as recommended by Hatchard and Parker. However, up to a concentration of $10^{-4} \mathrm{M}$, no influence on the quantum

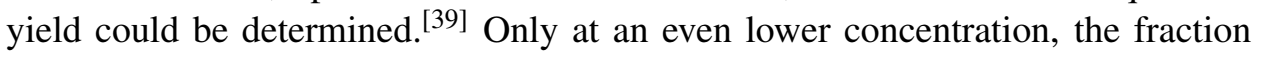


of oxalate-bound $\mathrm{Fe}^{\mathrm{III}}$ was reduced in favor of $\mathrm{Fe}^{\mathrm{III}}$-hexaaqua-, $\mathrm{Fe}^{\mathrm{III}}$-sulfato- and $\mathrm{Fe}^{\mathrm{III}}$-hydroxo complexes, which have different photochemical properties and reaction products than the ferrioxalate complex. ${ }^{[40]}$ The excess oxalate is dissolved as free $\mathrm{C}_{2} \mathrm{O}_{4}{ }^{2-}$.

But in contrast, many research groups report strongly deviating quantum yields at low concentrations of ferrioxalate already at the beginning of the irradiation process. $31 \mid 41-45]$ These inconsistencies finally motivated Weller et al. to further in-depth investigations. ${ }^{40]}$ Operating the ferrioxalate actinometer with flash photolysis and an identical number and wavelengths of the irradiated photons, the conversion dropped to about $14 \%$ compared to continuous irradiation. Implicationally, the deviation can be attributed to the temporal concentration of the reactive species. Flashlight splits off carboxyl radicals from the ferrioxalate complex within $20 \mathrm{~ns}$ according to reaction equations $\mathrm{R} 2.2$ and $\mathrm{R} 2.3$. Before the carboxyl radicals react further, micromolar concentrations of $\mathrm{CO}_{2}{ }^{\bullet-}$ are formed within very short time scales with rates in the order of $\mathrm{mol} \mathrm{s}^{-1}$. Under continuous irradiation, only rates in the order of nmol s${ }^{-1}$ are achieved, meaning that this process is several orders of magnitude slower. This difference in concentration causes different probabilities for the consecutive processes that follow the release of $\mathrm{CO}_{2}{ }^{\bullet-}$. The sensitivity of the continuous measurement and thus also the accuracy is obviously much higher.

With respect to $\mathrm{Fe}^{\mathrm{II}}$, the described quantum yield of about 1.2 can only be reached if there is a fraction of $70 \%$ unphotolyzed metal complexes in the immediate vicinity for an unhindered secondary reaction $\mathrm{R} 2.5$, [40] Weller et al. found that in a $1 \mathrm{~cm}$ cuvette, this condition is no longer valid below a concentration of $5 \cdot 10^{-3} \mathrm{M}$. Between $5 \cdot 10^{-4}$ and $5 \cdot 10^{-5} \mathrm{M}$, the rate is slowed down to finally completely stop at a concentration of less than $5 \cdot 10^{-6} \mathrm{M}$. This finding was confirmed with the help of femtosecond infrared spectroscopy. After the excitation of the ferrioxalate molecule and the cleavage of $\mathrm{CO}_{2}$ (reaction $\mathrm{R} 2.2$ ), the carboxyl radical is cleaved off (reaction R 2.3). 29

According to reaction equation $\mathrm{R} 2.4$ the carboxyl radical and the $\mathrm{CO}_{2}$ molecule are in local equilibrium with the oxalyl radical $\left(\mathrm{C}_{2} \mathrm{O}_{4}\right)^{\bullet-}$. The latter, however, could not be shown to be reactive towards an unexcited ferrioxalate complex, so that $\left(\mathrm{CO}_{2}\right)^{\bullet-}$ is exclusively responsible for the overall quantum yield. [27/28] At this stage, the consecutive reactions of attack on an unreacted ferrioxalate complex (reaction $\mathrm{R} \mathrm{2.5}$ p or recombination to an oxalate anion (reaction $\mathrm{R} \mathrm{2.6}$ ) are in competition. 40

$$
\left(\mathrm{CO}_{2}\right)^{\bullet-}+\left(\mathrm{CO}_{2}\right)^{\bullet-} \stackrel{1.2 \cdot 10^{9} \mathrm{~s}^{-1}}{\longrightarrow}\left(\mathrm{C}_{2} \mathrm{O}_{4}\right)^{2-}
$$

Reaction R 2.5 increases the quantum yield, whereas oxalate anions formed in reaction $\mathrm{R} 2.6$ have no influence on the quantum yield. A coulomb limit causes the small time constant for reaction R 2.5, so that this reaction takes place 1000fold slower than under pure diffusion limitation. $\stackrel{[29]}{ }$ The recombination according 
to reaction equation $\mathrm{R} 2.5$ is over 4000 times faster and thus is preferred in the case of a rapid increase of the carboxyl radical concentration. As a result, the apparent quantum yield decreases because of a lack of unexcited ferrioxalate complexes in the immediate vicinity.

Even under the original reaction conditions of Hatchard and Parker, the parallel reaction R 2.6 might be responsible for the upper limit of the experimentally observed maximum quantum yield of 1.26 for an irradiation wavelength above $254 \mathrm{~nm}$. An indication is that no effort, even providing adding additional $\mathrm{Fe}^{\mathrm{III}}$, can further increase this value. ${ }^{[40]}$ In subsequent studies, this theory was supported by molecular dynamics simulations and according spectroscopic findings. 46.47

Another effect that contributes to a lower quantum yield at intense radiation is a higher rate of the recombination of $\mathrm{CO}_{2}{ }^{\bullet-}$ and $\mathrm{CO}_{2}$, shifting the equilibrium according to reaction equation $\mathrm{R} 2.4$

Other than for carboxyl radicals, oxalyl radicals do not reduce ferrioxalate complexes as demonstrated by FT-IR, at least on very short time scales. ${ }^{[29]}$ In this way, additional $\mathrm{CO}_{2}{ }^{\bullet-}$ reacts off without the formation of $\mathrm{Fe}^{\mathrm{II}}$, also lowering $\Phi$.

The found behavior on short time scales at high excitation ratios agrees qualitatively with the experimentally observed deviations of continuous and discontinuous measurements. It is concluded that the occurring inaccuracy of ferrioxalate actinometry may arise from a drop of the quantum yield at high incident photon fluxes.

\subsection{Transfer and Impact on Experimental Application}

The key factor for a changing quantum yield is the ferrioxalate concentration. Weller et al. found a concentration of $5 \cdot 10^{-3} \mathrm{M}$ in a $1 \mathrm{~cm}$ cuvette as lower limit for a constant quantum yield. $\stackrel{40]}{ }$ With this knowledge, it is possible to design experiments that simulate reaction conditions that are prone to a changing quantum yield during the reaction progress.

A convenient setup for demonstration was found in two conventional cuvettes with path lengths of $1 \mathrm{~cm}$ and $0.5 \mathrm{~cm}$, irradiated by a single $365 \mathrm{~nm}$ LED. For both cuvettes, ferrioxalate was step-wisely irradiated in intervals of $0.5 \mathrm{~s}$ with permanent mixing and compared to continuous irradiation without mixing. These conditions represent the extreme cases of decent irradiation and adequate mixing versus intense irradiation with insufficient mixing. The determined conversions and photon fluxes are shown in Figure 6.

The conversion in the $1 \mathrm{~cm}$ cuvette is independent of the mixing. Linear regression yields a line of origin with hardly noticeable deviations up to conversions above $30 \%$ (Figure 6a, solid line). The conversions of the thoroughly mixed $0.5 \mathrm{~cm}$ cuvette also follow a straight line of origin, but show slightly larger deviations to both sides (dashed line). The conversion rate, as indicated by the slope, should be twice as high due to half the amount of substance, but this is not the case.

The data set of the not mixed $0.5 \mathrm{~cm}$ cuvette is strikingly different, initially showing an increase comparable to that of the thoroughly mixed cuvette, up to 


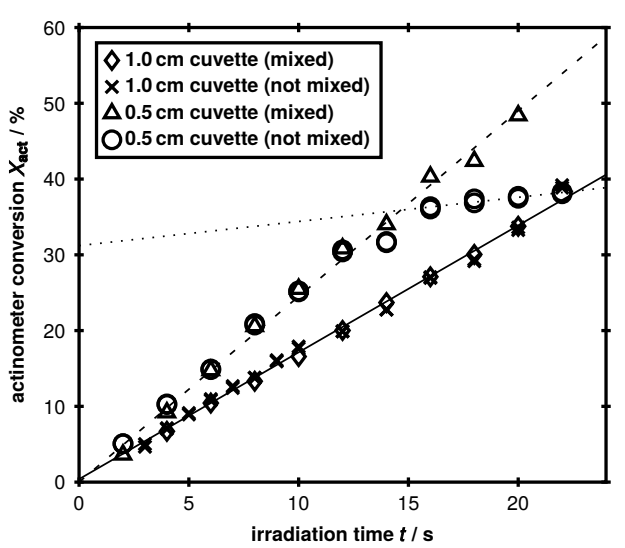

(a)



(b)

Figure 6: Actinometer conversion normalized on the same volume (a) and absorbed photon fluxes (b) upon irradiation of the $0.5 \mathrm{~cm}$ and $1 \mathrm{~cm}$ cuvette with and without permanent mixing.

about $37 \%$ conversion after $16 \mathrm{~s}$. During the further reaction progress, however, the conversion rate drops sharply. Despite this, the measured values can be approximated with a straight line in this range as well (dotted line). A y-axis intercept of approx. $32 \%$ occurs, showing a phenomenon analogous to Figure $1 \mathrm{a}$.

Converting the conversions to photon fluxes as shown in Figure 6b, the average $q_{\mathrm{abs}}$ for the $1 \mathrm{~cm}$ cuvette yields $1.4 \cdot 10^{-6} \mathrm{~mol} \mathrm{~s}^{-1}$ (solid line). The measured values for the non-mixed cuvette fluctuate noticeably around the mean value. In the mixed $0.5 \mathrm{~cm}$ cuvette, a $q_{\text {abs }}$ of about $1.0 \cdot 10^{-6} \mathrm{~mol} \mathrm{~s}^{-1}$ is measured (dashed line). For shorter irradiation times, even lower $q_{\mathrm{abs}}$ are detected. The $q_{\mathrm{abs}}$ determined from the individual measurements of the not mixed $0.5 \mathrm{~cm}$ cuvette cannot be meaningfully averaged with a horizontal line. While the values are at the level of the mixed experiment at the beginning, from about $12 \mathrm{~s}$ irradiation time a continuous decrease can be noticed.

Interpreting these findings with the insights described in the previous section, the concentration of ferrioxalate in the $1 \mathrm{~cm}$ cuvette is still sufficiently high. But without mixing, a loss of precision is already noticeable by the increased deviation. Otherwise, $\Phi$ is no longer constant in the $0.5 \mathrm{~cm}$ cuvette. Due to high local concentrations of $\left(\mathrm{CO}_{2}\right)^{\bullet-}$, a recombination occurs and the conversion of ferrioxalate remains limited to finally yield a wrong $q_{\mathrm{abs}}$. Even more remarkable, a linear approximation of the measured values from $16 \mathrm{~s}$ to $22 \mathrm{~s}$ together with a shift of the $\mathrm{y}$-axis intercept leads to a much too low $q_{\text {abs }}$ of $0.13 \cdot 10^{-6} \mathrm{~mol} \mathrm{~s}^{-1}$ (dotted line). These results on the one hand proof that high photon fluxes cause the observed inconsistencies for low ferrioxalate concentrations by increasing the probability of carboxyl radical recombination. On the other hand, these results show that enhanced mass transport helps to overcome this limitation. 
Consequently, the differences between the continuous and discontinuous measurements in the different capillary photoreactors discussed above are a result of the high local photon flux incident on the reactor. During discontinuous operation, a reduction of the local concentration of the carboxyl radicals can only occur by recombination, finally leading to a reduced quantum yield of the ferrioxalate reduction. For continuous operation, mass transport processes help to keep the local concentrations of carboxyl radicals low. This maintains a high quantum yield.

\section{Conclusion}

Motivated by experimental inconsistencies of ferrioxalate actinometer measurements, the drop of the quantum yield under high incident photon fluxes was identified by combining own experimental results and recent spectroscopic findings. High photon fluxes lead to extended local excitation of ferrioxalate complexes during short periods and with this to high local radical concentrations. If mass transport is too slow, the recombination reaction is preferred.

Generally concluding on ferrioxalate actinometry, insufficient mixed, stop-flow or discontinuous actinometry measurements can show inconsistencies due to a changing quantum yield. Such results are not eligible for further analysis and interpretation. To compensate for this, continuous measurements are recommended if possible, or at least a sufficient mixing must be ensured in intensely irradiated photoreactors. Increasing the ferrioxalate concentration can only encounter the problem partially since the risk of precipitation during the irradiation process increases. If the experimental conditions inevitably exceed the limits of the actinometer method, alternatives like radiometry should be considered. 


\section{Experimental Section}

\subsection{Reactor Models and Irradiation Devices}

The reactors consisted of a 3D-printed acrylonitrile butadiene styrene (ABS) scaffold to hold fluorinated ethylene propylene (FEP) capillaries with an outer diameter of $1 / 8$ " and an inner diameter of 1/16" (Figure 7a). While the inner volume of a whole capillary was $3 \mathrm{~mL}$, the actually irradiated volume was between 0.7 and $1.0 \mathrm{~mL}$ depending on the specific arrangement.

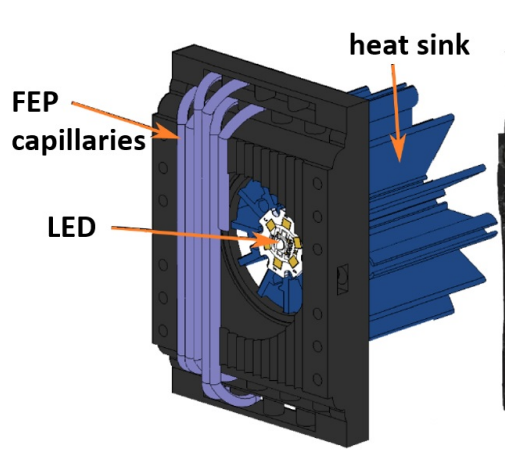

(a)

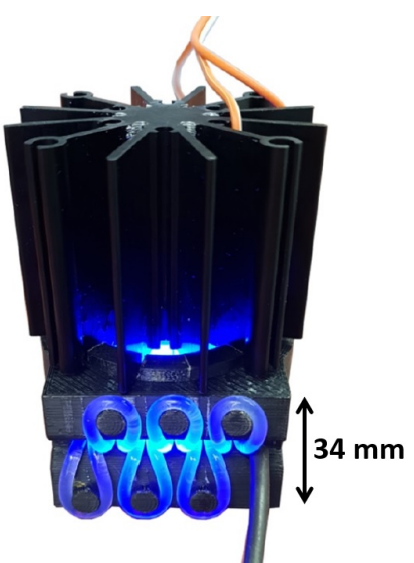

(b)

Figure 7: View on the scaffold 3D-printed from ABS and the irradiated FEP capillaries in the direction of the LED emission (a) and in operation while irradiated with the LED mounted on a heat sink (b).

A $405 \mathrm{~nm}$ LED NVSU233A(T)-D1 from NICHIA was mounted on a metal heat sink that can be precisely positioned in the center of the reactor basis. The LED was operated with an electrical current of $700 \mathrm{~mA}$. The setup in operation is shown in Figure 7b. Geometrically, an LED emission angle of $115^{\circ}$ hits the capillary surface.

\subsection{Experimental Setup}

To perform the pumped experiments or fill the capillaries with the $0.16 \mathrm{M}$ ferrioxalat solution, an ISMATEC RH1 CKC rotary piston pump was used. Recycling experiments were conducted with a total volume of $20 \mathrm{~mL}$ in a stirred storage vessel and a flow rate of $20 \mathrm{~mL} \mathrm{~min}^{-1}$. A scheme of the overall setup is shown in Figure 8 


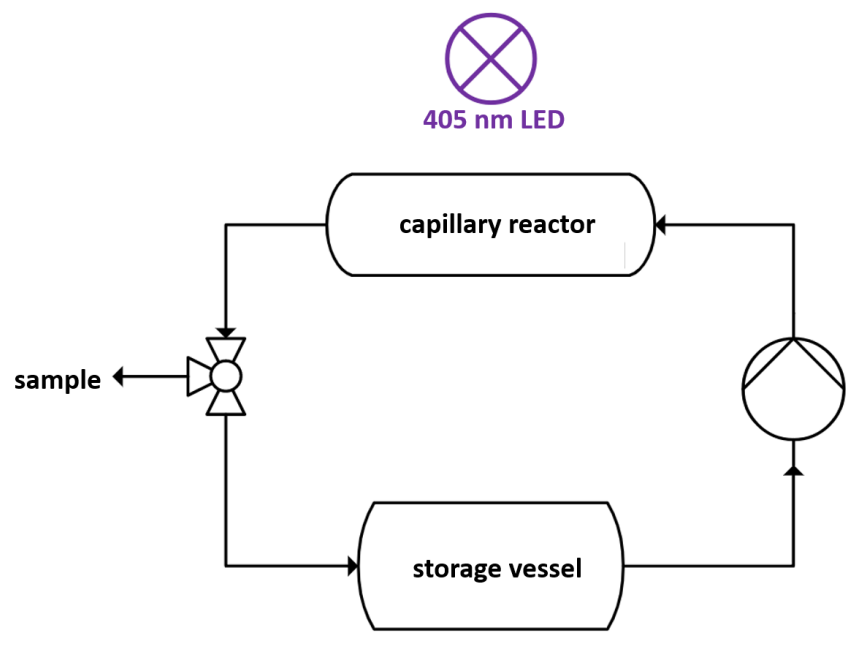

Figure 8: Schematic setup for recycle measurements including the irradiation unit of photoreactor and LED, the storage vessel, the pump and a spot to take samples.

\subsection{Parameters of the Actinometric Procedure}

Following irradiation in the reactor, two actinometer samples of $1 \mathrm{~mL}$ were diluted with $24 \mathrm{~mL}$ of $0.05 \mathrm{M}$ sulfuric acid in parallel. An aliquot of $5 \mathrm{~mL}$ from the diluted samples was developed by adding $15 \mathrm{~mL}$ of a $0.05 \mathrm{M}$ sulfuric acid solution containing $0.03 \mathrm{M}$ sodium acetate trihydrate and $0.003 \mathrm{M} \mathrm{1,10-phenanthroline.} \mathrm{After}$ $1 \mathrm{~h}$, the concentration of the samples was determined spectrometrically at $510 \mathrm{~nm}$ in triplets.

\subsection{Code Availability}

The Python code for evaluating the actinometric measurements, the used .qtifile and a description of the used reference measurement setup are available on GitHub https://github.com/photonZfeed/FerriOxActinometry and as a stable version on Zenodo https://doi.org/10.5281/zenodo.4849293. Version v1.0 was used for evaluation.

\section{Conflicts of interest}

There are no conflicts to declare.

\section{Acknowledgment}

The authors gratefully acknowledge the financial support provided by the German Research Foundation (ZI 1502/4-1). 


\section{References}

[1] D. Cambié, C. Bottecchia, N. J. W. Straathof, V. Hessel, T. Noël, Chem. Rev. 2016, 116, 10276-10341.

[2] Microreactors in Organic Synthesis and Catalysis, (Ed.: T. Wirth), Wiley$\mathrm{VCH}, 2013$.

[3] R. L. Hartman, J. P. McMullen, K. F. Jensen, Angewandte Chemie International Edition 2011, 50, 7502-7519.

[4] P. Seeberger, K. Geyer, T. Gustafsson, Synlett 2009, 2009, 2382-2391.

[5] M. M. Abdou, R. A. El-Saeed, S. Bondock, Arabian Journal of Chemistry 2019, 12, 88-121.

[6] G. Pritschow, Autonome Produktion, Springer Verlag, 2004.

[7] D. Ziegenbalg, B. Wriedt, G. Kreisel, D. Kralisch, Chemical Engineering \& Technology 2016, 39, 123-134.

[8] H. J. Kuhn, S. E. Braslavski, R. Schmidt, Pure and Applied Chemistry 2004, 76, 2105-2146.

[9] C. G. Hatchard, C. A. Parker, Admiralty Materials Laboratory 1956.

[10] A. Sugimoto, T. Fukuyama, Y. Sumino, M. Takagi, I. Ryu, Tetrahedron 2009, 65, 1593-1598.

[11] T. Aillet, K. Loubiere, O. Dechy-Cabaret, L. Prat, Chemical Engineering and Processing: Process Intensification 2013, 64, 38-47.

[12] D. Ziegenbalg, G. Kreisel, D. Weiß, D. Kralisch, Photochemical \& Photobiological Sciences 2014, 13, 1005-1015.

[13] Ü. Taştan, D. Ziegenbalg, Chemistry - A European Journal 2016, 22, 1882418832.

[14] M. Sender, D. Ziegenbalg, Chem. Ing. Tech. 2017, 89, 1159-1173.

[15] I. Reim, B. Wriedt, Ü. Tastan, D. Ziegenbalg, M. Karnahl, ChemistrySelect 2018, 3, 2905-2911.

[16] B. Wriedt, D. Kowalczyk, D. Ziegenbalg, ChemPhotoChem 2018, 2, 913921.

[17] Ü. Taştan, P. Seeber, S. Kupfer, D. Ziegenbalg, Reaction Chemistry \& Engineering 2021, 6, 90-99.

[18] F. Guba, F. Gaulhofer, D. Ziegenbalg, Journal of Flow Chemistry 2021.

[19] S. Triemer, M. Schulze, B. Wriedt, R. Schenkendorf, D. Ziegenbalg, U. Krewer, A. Seidel-Morgenstern, Journal of Flow Chemistry 2021.

[20] T. Aillet, K. Loubiere, O. Dechy-Cabaret, L. Prat, International journal of Chemical reactor Engineering 2014, 12, 1-13.

[21] H. A. Taylor, J. M. Fitzgerald, Analytical Photochemistry and Photochemical Analysis 1971, 91-115.

[22] W. D. Bowman, J. N. Demas, J. Phys. Chem. 1976, 80, 2434-2435.

[23] A. D. Kirk, C. Namasivayam, Anal. Chem. 1983, 55, 2428-2429.

[24] S. L. Murov, Handbook of Photochemistry, Vol. Sect. 13, Marcel Dekker, 1993. 
[25] L. Vincze, T. J. Kemp, P. R. Unwin, Journal of Photochemistry and Photobiology A: Chemistry 1999, 123.

[26] T. Lehóczki, É. Józsa, K. Ösz, Journal of Pure and Applied Chemistry A: Chemistry 2013, 521, 63-68.

[27] S. Straub, P. Brünker, J. Lindner, P. Vöhringer, Phys. Chem. Chem. Phys. 2018, 20, 21390-21403.

[28] S. Straub, P. Brünker, J. Lindner, P. Vöhringer, Angewandte Chemie International Edition 2018, 57, 5000-5005.

[29] F. H. Pilz, J. Lindner, P. Vöhringer, Physical Chemistry Chemical Physics 2019, 21, 23803-23807.

[30] J. H. Baxendale, N. K. Bridge, The Journal of Physical Chemistry 1955, 59, 783-788.

[31] E. Fernández, J. M. Figuera, A. Tobar, Journal of Photochemistry 1979, 11, 69-71.

[32] B. Wriedt, D. Ziegenbalg, Journal of Flow Chemistry 2020, 10, 295-306.

[33] A. F. M. Silvares, C. A. O. do Nascimento, E. Oliveros, S. H. Bossmann, A. M. Braun, Industrial \& Engineering Chemistry Research 2007, 46, 74367447.

[34] E. B. Corcoran, J. P. McMullen, F. Lévesque, M. K. Wismer, J. R. Naber, Angewandte Chemie International Edition 2020.

[35] M. Montalti, A. Credi, L. Prodi, M. T. Gandolfi, Handbook of Photochemistry, Taylor \& Francis, 3rd Edition, 2005.

[36] T. D. Svejstrup, A. Chatterjee, D. Schekin, T. Wagner, J. Zach, M. J. Johansson, G. Bergonzini, B. König, ChemPhotoChem 2021, DOI 10 . 1002/ cptc.202100059.

[37] J. N. Demas, W. D. Bowman, E. F. Zalewski, R. A. Velapoldi, J. Phys. Chem. 1981, 85, 2766-2771.

[38] J. Lee, H. H. Seliger, The Journal of Chemical Physics 1964, 40, 519-523.

[39] S. Goldstein, J. Rabani, J. Photochem. Photobiol. A 2008, 193, 50-55.

[40] C. Weller, S. Horn, H. Herrmann, Journal of Photochemistry and Photobiology A: Chemistry 2013, 255, 41-49.

[41] H. Funayama, K. Ogiwara, T. Sugawara, H. Ohashi, Kagaku Kogaku Ronbunshu 1984, 10, 446-453.

[42] L. Vincze, S. Papp, Journal of Photochemistry 1987, 36, 289-296.

[43] G. Duka, D. Batyr, L. Romanchuk, A. Sychev, Soviet Journal of Coordination Chemistry 1990, 16, 93-106.

[44] B. C. Faust, R. G. Zepp, Environmental Science \& Technology 1993, 27, 2517-2522.

[45] H. B. Abrahamson, A. B. Rezvani, J. Brushmiller, Inorganica Chimica Acta 1994, 226, 117-127.

[46] Y. Ogi, Y. Obara, T. Katayama, Y.-I. Suzuki, S. Y. Liu, N. C.-M. Bartlett, N. Kurahashi, S. Karashima, T. Togashi, Y. Inubushi, K. Ogawa, S. Owada, M. Rubešová, M. Yabashi, K. Misawa, P. Slaviček, T. Suzuki, Structural Dynamics 2015, 2, 034901. 
[47] D. M. Mangiante, R. D. Schaller, P. Zarzycki, J. F. Banfield, B. Gilbert, ACS Earth and Space Chemistry 2017, 1, 270-276. 


\section{Graphical Abstract}

Motivated by systematic measurement inconsistencies, ferrioxalate actinometry was experimentally investigated for the reason. A drop of the quantum yield at too high irradiation intensities was identified and explained in line with recent spectroscopic findings. Finally, recommendations for reliable application of ferrioxalate actinometry are given.

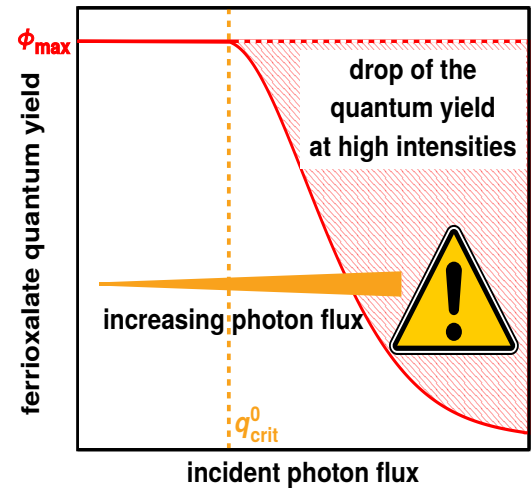

\section{Keywords}

Actinometry, Ferrioxalate, Microreactors, Modeling, Photochemistry, Photonic Efficiency. 


\title{
Application Limits of the Ferrioxalate Actinometer Supporting Information
}

\author{
Benjamin Wriedt ${ }^{a}$ and Dirk Ziegenbalg ${ }^{* a}$ \\ ${ }^{a}$ Institute of Chemical Engineering, Ulm University, Albert-Einstein-Allee 11, \\ 89081 Ulm, Germany, Fax: +49 7315012 25700; Tel: +49 73150 25703; mail: \\ dirk.ziegenbalg@uni-ulm.de
}

\section{S1 Absorption Coefficient and Actinometer Calibration}

The absorption coefficient of ferrioxalate was determined multiple times in a photo spectrometer with a solution of $0.16 \mathrm{~mol} \mathrm{~L}^{-1}$ (Figure S1). The slope of the calibration curve monitoring the measured absorbance over $\mathrm{Fe}^{2+}$ concentration was found to be $10980 \mathrm{~L} \mathrm{~mol}^{-1}$. Both were in straight accordance to the literature. [1] [3]

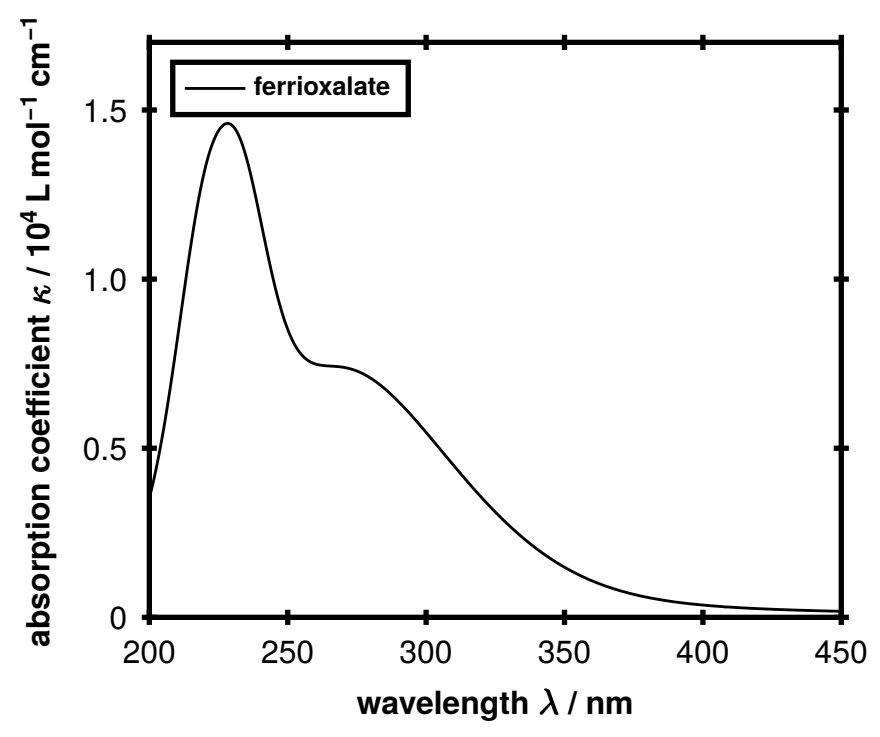

Figure S1: Measured absorption coefficient of ferrioxalate.

\section{S2 Continuous Measurement at Different Flow Rates with Increased Volume}

Increasing the total circulated volume from $20 \mathrm{~mL}$ to $40 \mathrm{~mL}$ at different flow rates does not show a significant deviation between the two variants. 


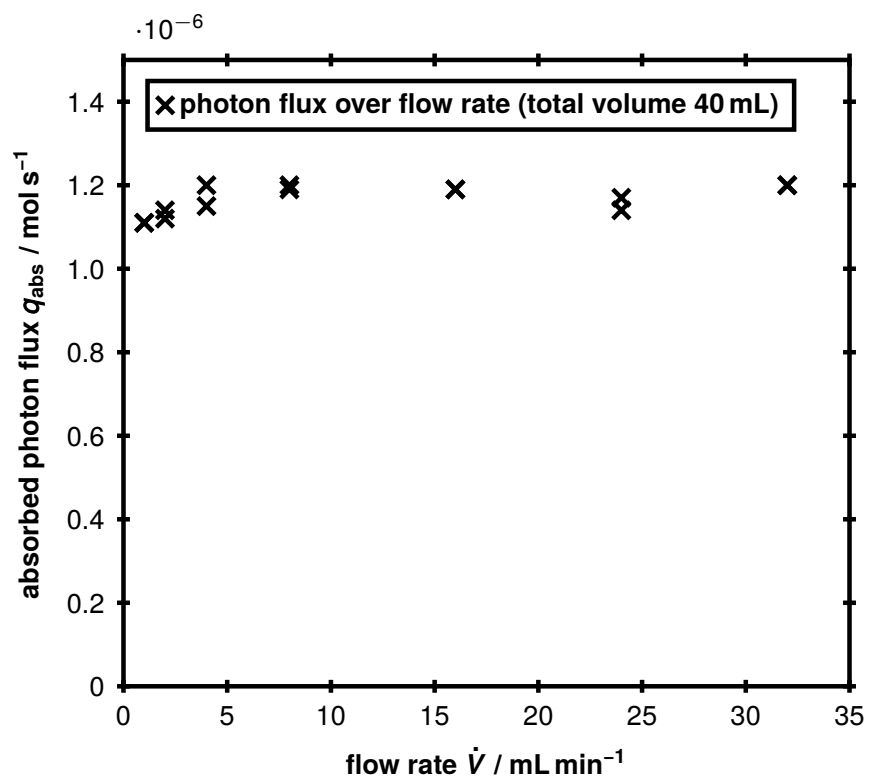

Figure S2: Test for an influence of increasing the total circulated volume from $20 \mathrm{~mL}$ (Figure $4 \mathrm{~b}$ ) to $40 \mathrm{~mL}$.

\section{References}

[1] M. Montalti, A. Credi, L. Prodi, M. T. Gandolfi, Handbook of Photochemistry, Taylor \& Francis, 3rd Edition, 2005.

[2] H. J. Kuhn, S. E. Braslavski, R. Schmidt, Pure and Applied Chemistry 2004, 76, 2105-2146.

[3] C. G. Hatchard, C. A. Parker, Admiralty Materials Laboratory 1956. 